\title{
Evaluación del estado trófico de tres cuencas interiores de Cataluña (Foix, Besòs y Llobregat) mediante la vegetación acuática: aplicación de un índice trófico (IVAM-FBL)
}

\author{
Jose Luis Moreno ${ }^{1 . *}$, Jorge De las Heras ${ }^{1}$, Narcís Prat ${ }^{2}$ y María Rieradevall ${ }^{2}$ \\ ${ }^{1}$ Centro Regional de Estudios del Agua (CREA), Ctra. de las Peñas, km 3, Albacete, 02071 \\ 2 Departament d'Ecologia, Universitat de Barcelona, Diagonal, 645, Barcelona 08028 \\ *Corresponding author: joseluis.moreno@uclm.es
}

Recibido: 13/7/07

Aceptado: 15/10/07

\begin{abstract}
Assessment of the trophic state of three inner basins in Cataluña (Foix, Besòs y Llobregat) through the aquatic vegetation: application of a trophic index (IVAM-FBL)

During April, 2005 a survey of the macroscopic aquatic vegetation was carried out in the basins of the Foix, Llobregat, and Besòs rivers with a total of 68 sampling stations. The main objective was to assess the trophic status of those basins by means of a Spanish macrophyte index called IVAM (Macroscopic Aquatic Vegetation Index). Forty-four taxa were identified: 8 genera of Cyanophyta, 5 of Rodophyta, 2 of Xantophyceae, 3 of Bacillariophyceae, 15 of Clorophyta, 8 of Spermatophyta, 1 lichen, and 2 bryophytes (mosses and liverworts). The Llobregat basin was the most diverse (37 taxa), followed by Besòs (33) and Foix (25). The most common taxa were Cladophora, Vaucheria, Oedogonium, Apium, Rorippa and Oscillatoria, along with mosses. Phanerogams were scarce, with Ranunculus, Zannichellia, and Potamogeton as the most common genera. The taxa with the highest IVAM score corresponding to good water quality (tolerance value, $\mathrm{vt}=8$ ) were, although scarce, Ranunculus, Cymbella, Rivularia, Nostoc, Tolypothrix, Chroococcus, and Lemanea, which indicate oligotrophic conditions. In good quality waters, although slightly eutrophic (mesotrophic waters vt $=6$ ), the most frequent taxa were Chara, Monostroma, and Hildenbrandia, along with liverworts and one lichen. According to the trophic qualifications provided by the IVAM index, $38.2 \%$ of sites had a good trophic status (classes I and II) while $61.8 \%$ of sites had an altered status. The IVAM response to nutrient concentration (phosphate, ammonium, nitrite, and nitrate) was always significant and a high correlation between IVAM and phosphate concentration $(r=0.71, p<0.001)$ can be highlighted, with a value higher than those reached by other diatom and macrophyte indices that use a species-level taxonomic resolution. This supports the advantage of using the IVAM index in the trophic status assessment of rivers since it is not necessary to reach a taxonomic species level to obtain a good response to this kind of disturbance.
\end{abstract}

Key words: Macrophytes, macroalgae, biotic index, eutrophication, Catalonia, Spain.

\section{RESUMEN}

Evaluación del estado trófico de tres cuencas interiores de Cataluña (Foix, Besòs y Llobregat) mediante la vegetación acuática: aplicación de un índice trófico (IVAM-FBL)

Durante abril de 2005 se realizó una campaña de muestreo de la vegetación acuática macroscópica en las cuencas de los ríos Foix, Besòs y Llobregat, sumando un total de 68 estaciones de muestreo. El objetivo principal fue evaluar el estado trófico de dichas cuencas mediante el índice trófico generado en España denominado IVAM (Índice de Vegetación Acuática Macroscópica). Se identificaron un total de 44 táxones, 8 géneros de Cyanophyta, 5 de Rodophyta, 2 de Xantophyceae, 3 de Bacillariophyceae, 15 de Clorophyta, 8 de Spermatophyta, 1 liquen y 2 briófitos (musgos y hepáticas). La cuenca del Llobregat fue la más diversa (37 táxones), seguida del Besòs (33) y el Foix (25). Los táxones más frecuentes fueron Cladophora, Vaucheria, Oedogonium, Apium, Rorippa y Oscillatoria, además de los musgos. Las fanerógamas fueron escasas, destacando los géneros Ranunculus, Zannichellia y Potamogeton. Los táxones que alcanzaron una mayor puntuación del IVAM (valor de tolerancia, $v t=8$ ), aunque poco frecuentes, fueron Ranunculus, Cymbella, Rivularia, Nostoc, Tolypothrix, Chroococcus $y$ Lemanea, todos indicadores de aguas oligotróficas. En aguas de buena calidad pero algo eutrofizadas (aguas mesotróficas, $v t=6$ ), los táxones más frecuentes fueron Chara, Monostroma y Hildenbrandia, además de las hepáticas y un liquen. Según la 
calificación de los tramos en clases de estado trófico realizada por el IVAM, el $38.2 \%$ de los tramos presentaron una buena calidad (clases I y II) mientras que el $61.8 \%$ presentaron un estado trófico alterado (clases III, IV y V). La respuesta del IVAM a la concentración de nutrientes (fosfato, amonio, nitrito y nitrato) fue siempre significativa, destacando la alta correlación alcanzada con el ión fosfato $(\mathrm{r}=0.71, \mathrm{p}<0.001)$, superior a la alcanzada por otros índices de diatomeas y macrófitos de resolución taxonómica específica. Este hecho confirma la ventaja de utilizar el IVAM en la evaluación del estado trófico de los ríos, ya que sin necesidad de alcanzar un nivel taxonómico de especie se obtiene una respuesta adecuada a dicha perturbación.

Palabras clave: Macrófitos, macroalgas, índice biótico, eutrofización, Cataluña, España.

\section{INTRODUCCIÓN}

La Directiva Marco del Agua (DMA, Directiva 2000/60/CE del Parlamento Europeo y del Consejo), contempla la flora acuática, junto con los invertebrados bentónicos y los peces, como un indicador biológico necesario para evaluar el estado ecológico de los ríos. La flora acuática comprende una amplia variedad de organismos microscópicos y macroscópicos, pertenecientes a grupos taxonómicos tan diferentes como las algas, los briófitos, los pteridófitos, los líquenes o las fanerógamas. Estos grupos han sido utilizados independientemente o en combinación para evaluar el estado trófico de una masa de agua. Desde hace dos décadas, en Europa se vienen utilizando los macrófitos acuáticos para evaluar la calidad del agua, comprobándose, en concreto, la utilidad de los índices tróficos basados en macrófitos en la evaluación del estado trófico de los ríos (Thiebaut et al., 2002). Sin embargo, son los micrófitos, y en concreto las diatomeas, el grupo de flora acuática más utilizado actualmente como bioindicador para la evaluación del estado ecológico ríos ibéricos, a través del cálculo de diversos índices europeos (p. ej. IPS: CEMAGREF, 1982; IBD: Prygiel y Coste, 1999; CEE: Descy y Coste, 1990). Dichos índices tienen el inconveniente de requerir una formación muy especializada para su aplicación, ya que se necesita realizar una determinación específica de los organismos.

En Europa se aplican actualmente varios índices que utilizan los macrófitos como indicadores del estado trófico de los ríos. Entre ellos, destacan: Mean Trophic Rank (MTR; Dawson et al., 1999) en Reino Unido, Trophic Index of
Macrophytes (TIM; Schneider y Melzer, 2003) en Alemania, y el Indice Biologique Macrophytique en Rivières (IBMR; AFNOR, 2003) junto con su antecesor el Scientific Group GIS Index (GIS; Haury et al., 1996, 2000) en Francia. Excepto el TIM, donde el nivel de determinación taxonómica exigido es el específico, los otros dos índices utilizan principalmente dos niveles de resolución: genérico y específico para algas, y específico para fanerógamas, briófitos y pteridófitos. En Cataluña, se han empleado algunos índices de macrófitos para evaluar la calidad del agua en algunas cuencas, destacando el trabajo realizado para la Agencia Catalana del Agua (ACA) en los ríos Francolí y Ter (ACA, 2003). En dicho trabajo, se han empleado tres índices de macrófitos: SLA (Sládecek y Sladeckova, 1996), SAP (Índice de Saprobios, Wegl, 1983) y E/P-I (Índice de Eutrofización-Polución, Dell'Uomo, 1991), índices que también requieren la determinación específica de los organismos en la mayoría de los casos. En los ríos Foix, Besós y Llobregat se han aplicado índices de diatomeas para evaluar la calidad del agua (ACA, 2003; Gomá et al., 2004), pero hasta ahora no existen estudios publicados en revistas científicas sobre la aplicación de índices de macrófitos en dichas cuencas. Sin embargo, en Cataluña está siendo aplicado el IVAM por diversos organismos gestores como la Agencia Catalana del Agua y la Confederación Hidrográfica del Ebro.

El objetivo principal del presente estudio es aplicar el IVAM para evaluar el estado trófico de las tres cuencas catalanas estudiadas, creando una nueva versión del índice adaptada a las condiciones tróficas locales. 
Foix

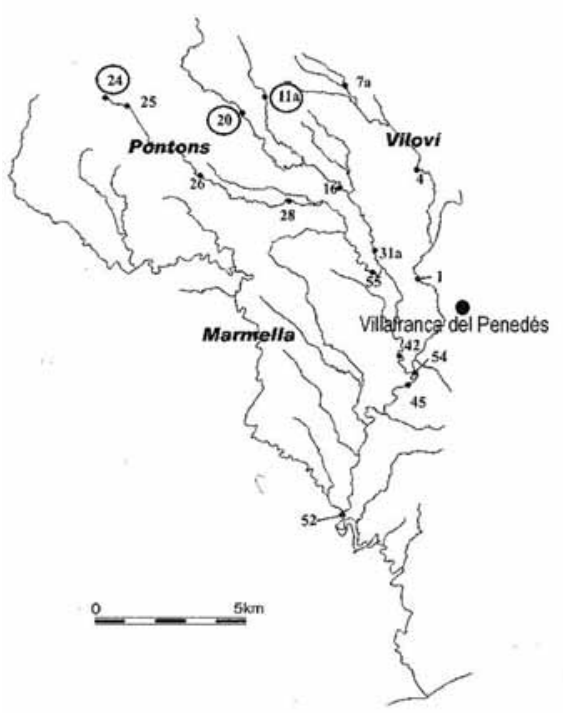

Llobregat

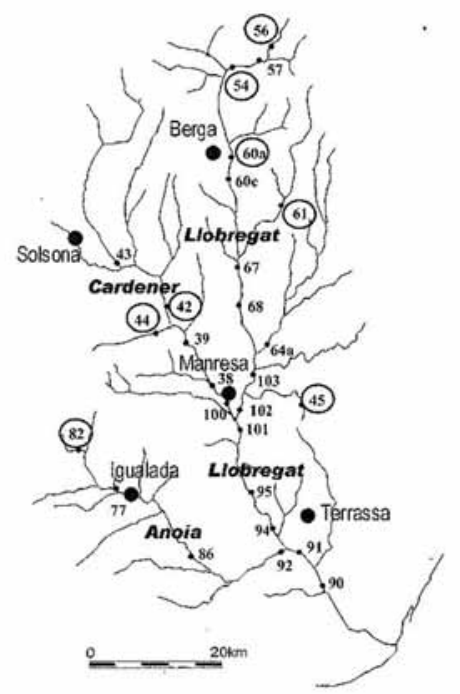

BESÒS

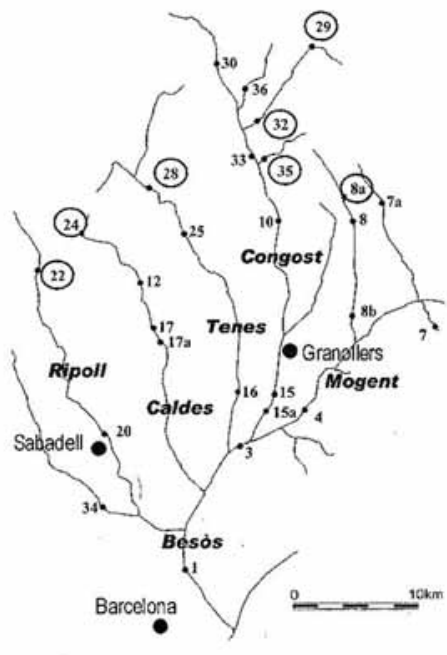

Figura 1. Localización de la zona de estudio indicando las principales subcuencas (en negrita cursiva) y puntos de muestreo (en circulo las "estaciones de referencia"). Location of the study area showing the main sub-basins (in italic bold) and sample sites (the circle denotes "reference station").

\section{METODOLOGÍA}

\section{Campaña de muestreo}

Durante abril de 2005 se realizó un muestreo de flora acuática en un total de 68 puntos de muestreo en tres cuencas interiores catalanas $\left(6424 \mathrm{~km}^{2}\right): 27$ en la cuenca del Besòs, 14 en el Foix, y 27 en el Llobregat (Fig. 1). En cada tramo se realizó un recorrido río arriba de unos $100 \mathrm{~m}$ cubriendo los hábitats presentes, tanto lóticos como leníticos (rápidos, pozas, orillas, etc.). Se recogió cualquier autótrofo acuático reconocible a simple vista, ya sea sumergido, semisumergido o flotante, lo que incluyó, según la terminología de Margalef (1983), tanto a micrófitos (sobretodo algas que forman comunidades de plocon y pecton) como a macrófitos (haptófitos, rizófitos y helófitos sumergidos). Los organismos recogidos pertenecieron a los siguientes grupos: algas, briófitos, líquenes y fanerógamas. Las muestras se tomaron con la mano, navaja o manga, fijadas en formaldehido $4 \%$ y determinadas bajo lupa y microscopio a nivel genérico excepto musgos, hepáticas y líquenes. En el campo se asignó a cada taxon un valor de abundancia en el tramo de 1 a 3 (raro, frecuente y abundante).

Con el objetivo de evaluar el estado trófico, se tomaron al mismo tiempo muestras de agua para el posterior análisis de nutrientes mediante métodos estandarizados (APHA, 1998): amonio (método Nessler), nitrito (método fotométrico), nitrato (cromatografía iónica) y fosfato (método colorimétrico del ácido ascórbico). La evaluación de la calidad de los tramos también incluyó la recogida de muestras de invertebrados siguiendo los correspondientes protocolos requeridos para la obtención de dos índices bióticos: IBMWP y su correspondiente IASPT (AlbaTercedor y Sánchez-Ortega, 1988; Alba-Tercedor et al., 2002) y FBILL (Prat et al., 1999).

\section{Obtención del IVAM-FBL}

A partir de los valores máximos de concentración de amonio y fosfato, se calcularon el valor de tolerancia (vt) y el valor indicador (vi) para cada taxon (Tabla 1), siguiendo la metodología propues- 
Tabla 1. Puntuaciones o valores de tolerancia (vt) y valor indicador (vi) para cada taxon considerado en el IVAM-FBL. Los asteriscos indican un cambio en el valor de tolerancia de los taxones respecto al IVAM-CLM. En negrita, taxones no contemplados en el IVAM-CLM. Tolerance scores and indicator values for the IVAM-FBL taxa. Asterisks denote changes in taxa tolerance values respect to the index IVAM-CLM. In bold, new taxa not recorded in the index IVAM-CLM.

\begin{tabular}{|c|c|c|c|}
\hline & & vt & vi \\
\hline \multicolumn{4}{|c|}{ Algas } \\
\hline \multicolumn{4}{|c|}{ Cyanophyta } \\
\hline 1 & Chroococcus & 8 & 2.5 \\
\hline 2 & Lyngbya* & 6 & 2.5 \\
\hline 3 & Microcoleus* & 2 & 1.0 \\
\hline 4 & Nostoc* & 8 & 2.5 \\
\hline 5 & Oscillatoria & 2 & 1.0 \\
\hline 6 & Rivularia* & 8 & 2.5 \\
\hline 7 & Schizothrix* & 6 & 2.5 \\
\hline 8 & Tolypothrix* & 8 & 2.5 \\
\hline \multicolumn{4}{|c|}{ Rhodophyta } \\
\hline 9 & Audouinella & 4 & 1.5 \\
\hline 10 & Batrachospermum* & 6 & 2.5 \\
\hline 11 & Compsopogon & 4 & 1.5 \\
\hline 12 & Hildenbrandia & 6 & 2.0 \\
\hline 13 & Lemanea* & 8 & 2.5 \\
\hline \multicolumn{4}{|c|}{ Heterocontophyta/Xanthophyceae } \\
\hline 14 & Tribonema* & 2 & 1.0 \\
\hline 15 & Vaucheria & 4 & 1.5 \\
\hline \multicolumn{4}{|c|}{ Heterokontophyta/Bacillariophyceae } \\
\hline 16 & Cymbella & 8 & 2.5 \\
\hline 17 & Gomphonema & 6 & 2.5 \\
\hline 18 & Melosira & 6 & 2.0 \\
\hline \multicolumn{4}{|c|}{ Clorophyta } \\
\hline 19 & Cladophora & 2 & 1.0 \\
\hline 20 & Chaetophora* & 6 & 2.5 \\
\hline 21 & Chara* & 6 & 2.5 \\
\hline 22 & Draparnaldia* & 6 & 2.5 \\
\hline 23 & Enteromorpha* & 6 & 2.5 \\
\hline 24 & Microspora* & 6 & 2.5 \\
\hline 25 & Monostroma* & 6 & 2.5 \\
\hline 26 & Mougeotia* & 6 & 2.5 \\
\hline 27 & Oedogonium & 2 & 1.0 \\
\hline 28 & Rhizoclonium & 2 & 1.0 \\
\hline 29 & Spirogyra & 2 & 1.0 \\
\hline 30 & Stigeoclonium & 2 & 1.0 \\
\hline 31 & Tetraspora* & 2 & 1.0 \\
\hline 32 & Ulothrix* & 4 & 1.5 \\
\hline 33 & Zygnema* & 2 & 1.0 \\
\hline \multicolumn{4}{|c|}{ Ascomycota (Líquenes) } \\
\hline 34 & Liquen & 6 & 2.0 \\
\hline
\end{tabular}

Tabla 1. (continuación)

\begin{tabular}{llll}
\hline \multicolumn{2}{c}{ Bryophyta } & vt & vi \\
\hline 35 & Musgos* & 4 & \\
36 & Hepáticas* $^{*}$ & 6 & 1.5 \\
\hline \multicolumn{4}{c}{ Spermatophyta (Fanerógamas) } \\
\hline 37 & Apium $^{*}$ & 2 & 2.5 \\
38 & Berula $^{*}$ & 2 & 1.0 \\
39 & Lemna $^{*}$ & 6 & 1.0 \\
40 & Potamogeton $^{*}$ & 4 & 2.0 \\
41 & Ranunculus $^{*}$ & 8 & 1.5 \\
42 & Rorippa $^{*}$ & 2 & 2.5 \\
43 & Veronica $^{*}$ & 2 & 1.0 \\
44 & Zanichellia $^{4}$ & 6 & 1.0 \\
\hline
\end{tabular}

ta por Moreno et al. (2005, 2006). Para la asignación del valor de tolerancia, se establecieron cuatro niveles de estado trófico correspondientes a cuatro valores de tolerancia:

- $v t=8$ (Grupo I): $\mathrm{P}_{-} \mathrm{PO}_{4}^{-3}<0.1 \mathrm{mg} / \mathrm{l}$, $\mathrm{N}-\mathrm{NH}_{4}^{+}<0.2 \mathrm{mg} / \mathrm{l}$

- $v t=6$ (Grupo II): $\mathrm{P}_{-} \mathrm{PO}_{4}^{-3} \quad 1-0.1 \mathrm{mg} / \mathrm{l}$, $\mathrm{N}-\mathrm{NH}_{4}^{+}<1 \mathrm{mg} / \mathrm{l}$

- $v t=4$ (Grupo III): $\mathrm{P}_{-} \mathrm{PO}_{4}^{-3}>1 \mathrm{mg} / \mathrm{l}$, $\mathrm{N}-\mathrm{NH}_{4}^{+}<1 \mathrm{mg} / \mathrm{l}$

- $v t=2$ (Grupo IV): $\mathrm{P}_{-} \mathrm{PO}_{4}^{-3}>1 \mathrm{mg} / \mathrm{l}$, $\mathrm{N}-\mathrm{NH}_{4}^{+}>1 \mathrm{mg} / \mathrm{l}$

Estos niveles han sido equiparados a la terminología utilizada en otros estudios sobre estado trófico de ríos e índices de macrófitos (p.ej. Haury et al., 2006; Schneider y Melzer, 2003): $\mathrm{vt}=8$ se ha considerado un nivel oligotrófico, $\mathrm{vt}=6$ mesotrófico, $\mathrm{vt}=4$ eutrófico $\mathrm{y} \mathrm{vt}=2$ hipertrófico o politrófico.

Igualmente, se calculó el valor indicador (vi) asignado a cada taxon el rango medido para ambos nutrientes $\left(\right.$ rango $\left.=x_{\text {máx }}-x_{\text {mín }}\right)$, de forma que el valor obtenido representa la amplitud trófica o euricidad de los táxones. Al igual que para el valor de tolerancia, se definieron 4 rangos de valores de nutrientes correspondientes a cuatro valores indicadores: 
- $v i=1.0: \mathrm{P}_{-} \mathrm{PO}_{4}^{-3}>1 \mathrm{mg} / \mathrm{l}, \mathrm{N}-\mathrm{NH}_{4}^{+}>1 \mathrm{mg} / \mathrm{l}$

- $v i=1.5: \mathrm{P}_{-} \mathrm{PO}_{4}^{-3}>1 \mathrm{mg} / \mathrm{l}, \mathrm{N}-\mathrm{NH}_{4}^{+}<1 \mathrm{mg} / \mathrm{l}$

- $v i=2.0: \mathrm{P}^{-} \mathrm{PO}_{4}^{-3} 1-0.2 \mathrm{mg} / \mathrm{l}, \mathrm{N}-\mathrm{NH}_{4}^{+}<$ $1 \mathrm{mg} / \mathrm{l}$

- $v i=2.5: \mathrm{P}_{-} \mathrm{PO}_{4}^{-3}<0.2 \mathrm{mg} / \mathrm{l}, \mathrm{N}-\mathrm{NH}_{4}^{+}<$ $1 \mathrm{mg} / \mathrm{l}$

El cálculo del índice se realizó aplicando la siguiente fórmula de medias ponderadas (Zelinka y Marvan, 1961):

$$
\operatorname{IVAM}=\frac{\sum v i_{i} c_{i} v t_{i}}{\sum v i_{i} c_{i}}
$$

donde $v i_{i}$ es el valor indicador del taxon $i$ que oscila entre 1 y $2.5 ; c_{i}$ es el valor de cobertura del taxon $i$, representado por tres valores: 1 $=<5 \%, 2=5-50 \%$ y $3=>50 \%$; y $v t_{i}$ el valor de tolerancia del taxon $i$, que varía entre $2 \mathrm{y}$ 8. Así, se obtuvo una adaptación del IVAM para las tres cuencas interiores catalanas, denominado IVAM-FBL (Foix, Besòs y Llobregat), que presenta algunos cambios en los valores de tolerancia e indicador respecto al original IVAM-CLM (Castilla-La Mancha), así como la inclusión de nuevos táxones y la exclusión de los táxones no presentes en dichas cuencas (Tabla 1).

Finalmente, y con objeto de crear 5 clases de calidad se seleccionaron los tramos escasamente perturbados en cuanto a concentración de nutrientes según estándares definidos para ríos mediterráneos peninsulares $\left(\mathrm{P}_{-} \mathrm{PO}_{4}^{-3}<0.05 \mathrm{mg} / \mathrm{l}\right.$, $\mathrm{N}-\mathrm{NH}_{4}^{+}<0.5 \mathrm{mg} / \mathrm{l}$, Bonada et al., 2002; $n=28$ ). Los intervalos (Tabla 2) se han obtenido calculando el percentil 75 de dichos tramos como valor mínimo de la clase I, y a continuación, tras restarle la menor puntuación posible en tramos con presencia de vegetación acuática (2), el intervalo resultante se dividió por 3 para obtener los límites entre el resto de clases (Moreno et al., 2006). Una modificación importante respecto a los rangos del IVAM-CLM, es considerar el valor 2 como clase V (anteriormente el valor 2 era considerado clase IV).
Tabla 2. Rangos y clases de calidad del IVAM-FBL. Range of values and quality types of IVAM-FBL.

\begin{tabular}{lcc}
\hline Estado Trófico & Clase & Rango \\
\hline Muy bueno & I & $>5.2$ \\
Bueno & II & $5.2-4.2$ \\
Moderado & III & $4.1-3.1$ \\
Deficiente & IV & $3.0-2.1$ \\
Malo & V & $\leq 2$ \\
\hline
\end{tabular}

Análisis estadísticos

El análisis de la composición florística se realizó mediante análisis cluster (método UPGMA, índice de similaridad de Jaccard), que agrupa las estaciones con una composición taxonómica más parecida, así como los táxones que presentan distribuciones más similares. El análisis fue realizado mediante el programa estadístico MVSP v3.11 (Multivariate Statistical Package, Kovach Computing Services, 1985-2000, http://www.kovcomp.com).

La respuesta al estado trófico (nutrientes) se evaluó mediante un análisis de correlación de Spearman (las variables no se ajustan a una distribución normal después de la correspondiente transformación) entre los valores obtenidos del IVAM-FBL y los índices de macroinvertebrados, y la concentración de nutrientes. Los análisis de correlación se realizaron mediante el paquete estadístico XLSTAT v3.4 (Thierry Fahmy, 19961998, http://www.xlstat.com).

\section{RESULTADOS}

\section{Composición florística, distribución de los táxones y niveles tróficos}

Se identificaron un total de 44 táxones (Tabla 1), de los cuales 33 géneros correspondieron a algas, 8 géneros a fanerógamas, 1 liquen y 2 briófitos (solo se diferenció entre dos tipos de briófitos: musgos y hepáticas). Los organismos más frecuentes, apareciendo en más de 10 sitios, fueron Cladophora (60 sitios), Vaucheria (36), Musgos (32), Oedogonium (29), Apium (28), Rorippa (26), Oscillatoria (24), Spirogyra (15), Hepáticas (11), Rhizoclonium (11) y Zygnema (11), mien- 
Tabla 3. Diversidad florística de las tres cuencas estudiadas (número de táxones IVAM registrados). Flower diversity of the three basins studied (number of IVAM taxa recorded).

\begin{tabular}{lccc}
\hline & Foix & Besòs & Llobregat \\
\hline briófitos & 2 & 2 & 2 \\
líquenes & 0 & 1 & 1 \\
cianofíceas & 3 & 6 & 6 \\
diatomeas & 2 & 2 & 2 \\
rodofíceas & 2 & 4 & 4 \\
xantofíceas & 2 & 2 & 2 \\
clorófitos & 10 & 11 & 13 \\
fanerógamas & 4 & 5 & 7 \\
\hline \multicolumn{1}{r}{ Total } & 25 & 33 & 37 \\
\hline
\end{tabular}

tras que los más raros, apareciendo en menos de tres sitios fueron Nostoc (2), Tolypothrix (2), Gomphonema (2), Batrachospermum (2), Chaetophora (2), Mougeotia (2), Lemna (2), Chroococcus (1), Lemanea (1), Enteromorpha (1) y Draparnaldia (1). La cuenca del Llobregat fue la más diversa con 37 táxones, seguida por la cuenca del Besòs con 33 y la del Foix con 25 (Tabla 3). Esta última presenta una baja diversidad de cianofíceas, rodofíceas y fanerógamas.

Con el objetivo de identificar grupos de táxones o comunidades indicadoras de niveles tróficos, se ha realizado un análisis cluster de la matriz de abundancia de especies y estaciones (Fig. 2). Se observa un gradiente trófico en el que los táxones indicadores de aguas de buena calidad se sitúan abajo, aumentando el nivel de eutrofia hacia la parte superior del dendrograma. Con el fin de definir niveles tróficos, se representa el valor medio de vt (valor de tolerancia) de los táxones que constituyen cada grupo identificado. Así, teniendo en cuenta que los 4 valores de tolerancia del IVAM $(2,4,6$ y 8) representan niveles tróficos (concentración de amonio y fosfato), éstos se pueden asignar, respectivamente, a aguas hipertróficas, eutróficas, mesotróficas y oligotróficas. A continuación se evalúa el estado de las tres cuencas en función de la distribución de los táxones y su valor como indicadores de niveles tróficos.

En las cabeceras silíceas de la cuenca del Besòs (Congost y Riera de Mogent), aparece una comunidad típica de cauces estrechos y sombreados que cubre las piedras en más de un $50 \%$, formada por un liquen verdoso no identificado y la

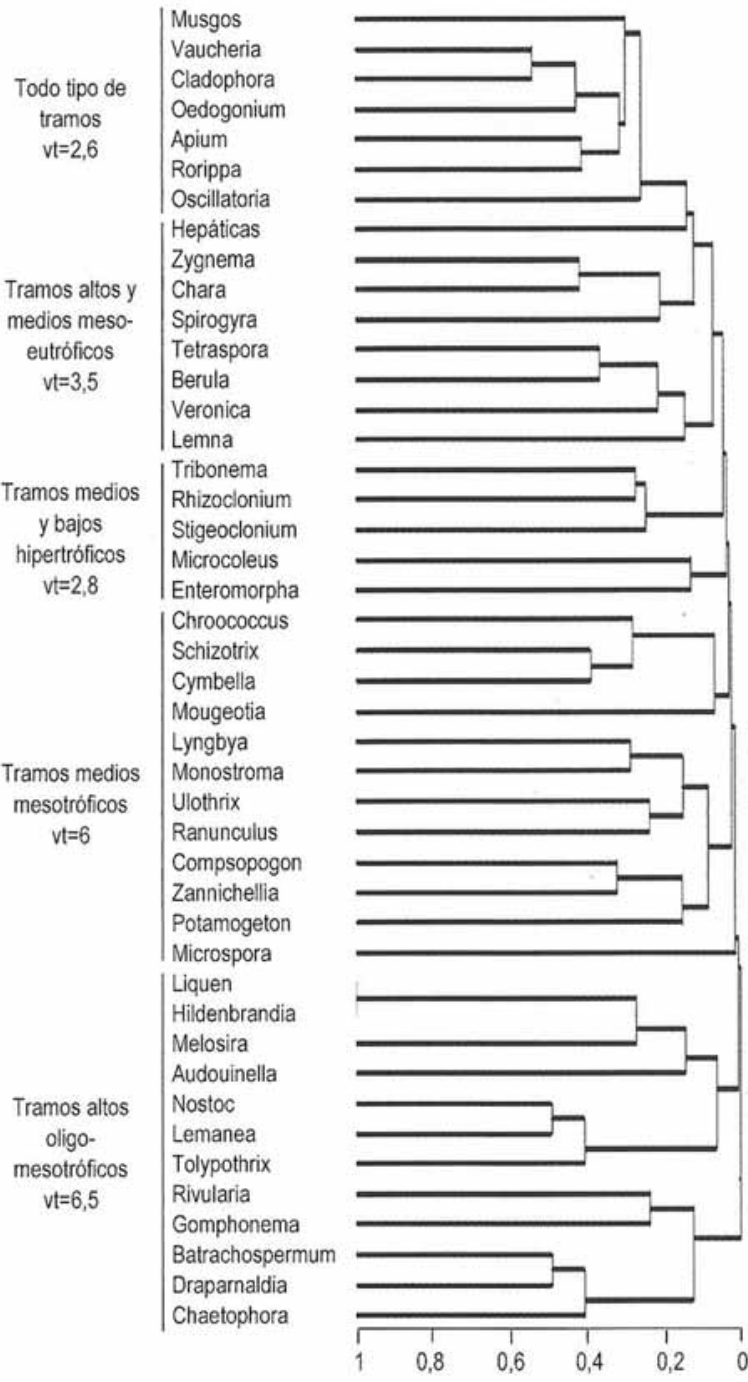

Figura 2. Análisis cluster de los táxones. La escala representa los valores del índice de Jaccard. Niveles tróficos definidos en función de los valores de tolerancia (vt) de los táxones. Se indica el valor medio de vt del grupo. Cluster analysis of the taxa. The scale represents the values of the Jaccard index. Trophic levels are defined according to taxa tolerance values (vt). The mean vt value per group is shown.

rodofícea Hildenbrandia (estaciones B32, B35, B8A, B8, B7A), constituyendo lo que podríamos calificar como una comunidad indicadora de tramos altos mesotróficos $(v t=6)$ (Tabla 1; Fig. 2). Es destacable la única presencia de Lemanea y Tolypothrix (B29) en las 3 cuencas estudiadas, constituyendo una comunidad de aguas oligotróficas $(v t=8)$. Otro género que apareció en 
solo una estación de litología granítica cubriendo más del $50 \%$ del lecho (B7) fue Draparnaldia, acompañada de Batrachospermum y Chaetophora (Fig. 2), comunidad indicadora de tramos mesotróficos $(\mathrm{vt}=6)$. Los tramos eutróficos e hipertróficos del Besòs se caracterizaron por una gran cobertura de Cladophora, con orillas cubiertas de Apium y Rorippa, encontrándose a menudo madejas mixtas de Tribonema, Rhizoclonium y Stigeoclonium $(\mathrm{vt}=2)$. Por último, citar la presencia en varias estaciones de la fanerógama Lemna en pozas marginales de arroyos de cabecera mesotróficos (B22, B28).

En cuanto a la cuenca del Llobregat, presentó 4 géneros de algas que no se han encontrado en las otras dos, destacando la presencia de Rivularia en 3 estaciones calcáreas oligotróficas de afluentes menores (L43, L44, L61) y de Monostroma en 9 estaciones de tramos medios calcáreos mesotróficos, al igual que dos géneros de fanerógamas: Ranunculus en 4 estaciones del tramo medio con cauce ancho y soleado del eje principal del Llobregat, de aguas oligomesotróficas (L60A, L60C, L67, L68), y Potamogeton, que aparece en 3 estaciones, dos de ellas de tramos bajos de aguas hipertróficas (L90, L91) y otra estación del Cardener de características mesotróficas (L42). Además de Potamogeton, los tramos hipertróficos estuvieron habitados principalmente por Cladophora (en numerosos casos cubriendo el 80-100\% del lecho), Vaucheria, Rhizoclonium, Oscillatoria y Stigeoclonium (Fig. 2). En arroyos bien conservados aparecen Rivularia, Chara y Zannichellia, comunidad indicadora de aguas mesotróficas.

La cuenca del Foix resultó ser la más pobre en vegetación acuática, debido principalmente a la alteración de sus cauces y la mala calidad de sus aguas por el impacto de la agricultura. La mayoría de las estaciones se pueden calificar de eutróficas o hipertróficas, caracterizadas por una alta cobertura de Cladophora y Vaucheria, acompañadas a veces por $R h i$ zoclonium y Stigeoclonium. Las cabeceras son calcáreas y se caracterizan por la abundancia de Hepáticas y Musgos (estaciones F7A, F11A, F20, F24, F28), acompañadas de Chara en algunas ocasiones y constituyendo una comunidad de aguas mesotróficas. La única fanerógama presente en los tramos visitados del Foix, a parte de los helófitos Rorippa, Apium y Veronica comunes en toda la cuenca, fue Zannichellia, solo presente en una única estación calcárea mesotrófica acompañada de Chara (F28).

Las masas mucilaginosas pardas o blanquecinas de diatomeas (Cymbella, Gomphonema) y Chrooccocus se encontraron en arroyos semisombreados de bajo caudal o formando charcas casi desconectadas, sobre roca madre y aguas claras (estaciones L44, L45, L61, B36), géneros que en el área de estudio indican aguas oligomesotróficas. En cuanto a las Zygnematofíceas, Zygnema tiende a aparecer más en lugares de referencia de mayor altitud que Spirogyra, y aunque a veces aparecen juntas, Spirogyra es más propia de tramos medios en el área de estudio. A menudo aparecen asociadas con Chara (Fig. 2). El género Oedogonium presentó una distribución muy variada, presentándose en un rango amplio de condiciones tróficas.

\section{IVAM-FBL: clases de calidad y nutrientes}

Se recogieron entre 1 y 12 táxones IVAM por punto, con un promedio de 6 táxones para las

Tabla 4. Valor medio $(x)$ y desviación típica (SD) del número de taxones IVAM-FBL, del IVAM-FBL y la concentración de nutrientes, para cada cuenca. Mean value $(\mathrm{x})$ and standard deviation (SD) of the number of IVAM-FBL taxa, IVAM-FBL index, and nutrient concentrations, for each basin.

\begin{tabular}{lrrrrrr}
\hline & Bèsos & \multicolumn{7}{l}{ Foix } & Llobregat & & & \\
\cline { 2 - 7 } $\mathrm{n}^{\text {o tramos }}$ estudiados & 27 & 14 & 27 & & & \\
\hline & $x$ & $\mathrm{SD}$ & $x$ & $\mathrm{SD}$ & $x$ & $\mathrm{SD}$ \\
\cline { 2 - 7 } $\mathrm{n}^{\text {o taxones }}$ & & & & & & \\
IVAM & 5.8 & 2.5 & 6.6 & 2.6 & 6.4 & 2.6 \\
IVAM-FBL & 3.3 & 1.4 & 3.4 & 0.9 & 3.8 & 1.2 \\
fosfato (mg/l) & 1.26 & 2.22 & 0.23 & 0.43 & 0.13 & 0.16 \\
amonio (mg/l) & 10.64 & 21.17 & 0.31 & 0.39 & 0.49 & 1.58 \\
nitrito (mg/l) & 0.12 & 0.16 & 0.24 & 0.32 & 0.04 & 0.07 \\
nitrato (mg/l) & 2.76 & 5.61 & 26.11 & 23.50 & 0.87 & 0.69 \\
& $n$ & $\%$ & $n$ & $\%$ & $n$ & $\%$ \\
\cline { 2 - 7 } tramos de las & & & & & & \\
clases I, II & 9 & 33.3 & 5 & 35.7 & 12 & 44.4 \\
tramos de las & & & & & & \\
clases III, IV, V & 18 & 66.7 & 9 & 64.3 & 15 & 55.6 \\
\hline
\end{tabular}


tres cuencas (Tabla 4). El número de táxones disminuyó en general con el incremento de la eutrofia y la altitud, aunque algunos puntos de mala calidad (clases IV y V), con valores altos de nutrientes, contaron con una elevada riqueza de táxones (entre 6 y 9), en este caso siempre tolerantes. Así, el mayor número de táxones, 12, se midió en estaciones de diferente calidad, clasificadas como de clase I (B36), II (L43) y III (F28). Según la calificación de los tramos en clases de estado trófico realizada por el IVAM-FBL (Tabla 4), el 38.2\% de los tramos estudiados presentaron un buen estado (clases I y II), mientras que el $61.8 \%$ presentaron un estado trófico alterado (clases III, IV y V). La cuenca del Llobregat fue la que presentó un mayor valor medio del IVAM-FBL (3.8) y un mayor porcentaje de tramos en buen estado (44.4\%).

Los valores más altos del IVAM-FBL correspondieron a las estaciones calificadas como "de referencia" según estudios previos (p. ej. Prat et al., 2003). El valor más alto del índice fue 6.18, medido en una estación de "referencia" de la cuenca del Besòs (B29), con solo 8 táxones. El máximo valor alcanzado en el Llobregat fue de 5.46 (7 táxones), también medido en una estación de referencia (L60A), mientras que en el Foix fue de 4.75 (5 táxones), clase II (F16, no de referencia). Excepto dos casos de estaciones de referencia en el Besòs (B28 y B22) y una en el Llobregat (L54), que fueron calificados por el IVAM-FBL como clase III, el resto de estaciones de referencia fueron calificadas de buena calidad (clases I y II).
Respecto a la concentración de nutrientes, son destacables los altos valores medidos en tramos calificados de baja calidad según el IVAM-FBL (clases IV y V). En concreto, las concentraciones de amonio en la cuenca del Besòs fueron muy altas (Tabla 4), mayores de $10 \mathrm{mg} / \mathrm{l}$ en las clases IV y V, debido probablemente a la abundancia de vertidos urbanos e industriales derivados de la alta densidad de población de la cuenca. Asimismo, la concentración media de fosfato fue mayor en el Besòs que en las otras cuencas. Los niveles de nitrato fueron llamativamente altos en la cuenca del Foix (Tabla 4), probablemente debido al desarrollo de la agricultura, principalmente viñedos. En esta cuenca, ninguna estación fue calificada por el IVAM-FBL como de clase I. El Llobregat fue la cuenca menos eutrofizada de las tres, debido probablemente a un mayor caudal de dilución. En oposición, en los tramos de las tres cuencas calificados como clase I y II, se midieron concentraciones de nutrientes bajas exceptuando el nitrato, que sobrepasó el valor de $1 \mathrm{mg} / \mathrm{l}$ en algunas ocasiones, sobretodo en el Besòs.

\section{Respuesta al estado trófico: correlaciones}

En la Tabla 5 se presentan los valores del coeficiente de correlación de Spearman entre el IVAM-FBL, otros índices bióticos y los nutrientes indicadores del estado trófico. Aunque todos los valores de correlación entre índices y nutrientes fueron significativos, destaca la alta correlación entre el IVAM-FBL y el fosfato $(r=-0.71, p<0.001)$, mayor que con cualquie-

Tabla 5. Valores de correlación de Spearman entre los índices y nutrientes. Se indican las correlaciones significativas $(*=p<0.05$, $* *=p<0.01 ; * * *=p<0.001)$. ns = no significativo. Spearman correlation values between indices and nutrients. Significant correlations are marked $(*=\mathrm{p}<0.05, * *=\mathrm{p}<0.01 ; * * *=\mathrm{p}<0.001) . n s=$ non significant.

\begin{tabular}{|c|c|c|c|c|c|c|c|c|c|}
\hline & unidades & IVAM-FBL & & IVAM-CLM & & IBMWP & & FBILL & \\
\hline IVAM-FBL & - & 1 & & & & & & & \\
\hline IVAM-CLM & - & 0.71 & $* * *$ & 1 & & & & & \\
\hline IBMWP & - & 0.76 & $* * *$ & 0.69 & $* * *$ & 1 & & & \\
\hline FBILL & - & 0.70 & $* * *$ & 0.71 & $* * *$ & 0.93 & $* * *$ & 1 & \\
\hline amonio & $\mathrm{mg} / \mathrm{l}$ & -0.41 & $* * *$ & -0.37 & $* *$ & -0.56 & $* * *$ & -0.44 & $* * *$ \\
\hline nitrito & $\mathrm{mg} / \mathrm{l}$ & -0.54 & $* * *$ & -0.53 & $* * *$ & -0.68 & $* * *$ & -0.66 & $* * *$ \\
\hline nitrato & $\mathrm{mg} / \mathrm{l}$ & -0.31 & $* *$ & -0.31 & $* *$ & -0.37 & $* *$ & -0.34 & $* *$ \\
\hline fosfato & $\mathrm{mg} / \mathrm{l}$ & -0.71 & $* * *$ & -0.65 & $* * *$ & -0.66 & $* * *$ & -0.64 & $* * *$ \\
\hline
\end{tabular}


ra de los otros índices considerados. Ello confirma una alta y adecuada respuesta del IVAM-FBL a la eutrofización, y su gran utilidad en la evaluación del estado trófico de los ríos catalanes. Sin embargo, los invertebrados (índices IBMWP y FBILL) respondieron mejor a las formas disueltas de nitrógeno (amonio, nitrito y nitrato) (Tabla 5). Estos resultados indican la utilidad tanto de los productores primarios como de los consumidores en la evaluación del estado trófico de los ríos (Moreno et al., 2005). Por otra parte, la adaptación del IVAM a las condiciones locales de las tres cuencas catalanas, ha resultado en una mayor respuesta a la eutrofización, como se puede comprobar comparando los valores de correlación entre IVAM-CLM y los nutrientes, que son menores que los obtenidos con el IVAM-FBL.

\section{DISCUSIÓN}

\section{Comparación con el IVAM-CLM}

El IVAM-FBL ha incluido un total de 44 táxones, frente a los 53 considerados para el IVAMCLM originado en Castilla-La Mancha (Moreno et al., 2005, 2006). Un total de 13 táxones presentes en Castilla-La Mancha no han sido encontrados en las tres cuencas catalanas, faltando 7 géneros de cianófitos (Cylindrospermum, Nodularia, Anabaena, Plectonema, Scytonema, Phormidium, Arthronema), 2 de clorófítos (Hydrodictyon, Nitella), 3 de espermatófitos (Callitriche, Ceratophyllum, Myriophyllum) y 1 pteridófito (Azolla). Sin embargo, 5 géneros de algas (Chroococcus, Compsopogon, Melosira, Cymbella, Gomphonema) y un líquen, que no estaban incluidos en el IVAM-CLM, han sido encontrados en Cataluña, destacando la inclusión de dos grupos nuevos: líquenes y diatomeas. Asimismo, los valores de tolerancia y/o indicador de 28 táxones han cambiado respecto a los calculados para el IVAM-CLM, debido a que las concentraciones de amonio y fosfato en las que fueron encontradas dichos táxones en Cataluña fueron diferentes a las de Castilla-La Mancha: en el caso de 11 táxones las concentraciones fueron menores, y en 14 casos fueron mayores, mientras que en 3 casos no cambiaron los valores de tolerancia pero sí los rangos de concentración (vi), que fueron menores. Este hecho corrobora el doble argumento de que los valores de tolerancia e indicador de los géneros pueden variar entre diferentes regiones o países (Moreno et al., 2006), y que se debe tener especial precaución a la hora de comparar valores de índices entre tipos de ríos diferentes (Dawson et al., 1999; Thiebaut et al., 2002). Sin embargo, el objetivo futuro del índice pasa por calcular un único valor de tolerancia e indicador para cada género teniendo en cuenta el máximo número de datos recogidos de diferentes cuencas ibéricas, de forma que exista un único índice IVAM aplicable a toda la Península.

En España, destaca la aplicación del IVAM en Castilla-La Mancha (IVAM-CLM) (Moreno et al., 2005, 2006). No existe ningún otro índice de macrófitos originado en España para evaluar la calidad de los ríos, a excepción de un índice aplicado en la cuenca del Segura denominado IM (Índice de Macrófitos, Suárez et al., 2005), que presenta varios inconvenientes: es un índice de naturaleza aditiva que prima la riqueza sobre el valor de tolerancia de los táxones; el nivel de resolución taxonómica es muy bajo y considera una escasa diversidad de táxones; proporciona unos valores de tolerancia de forma subjetiva (no son calculados a partir de la concentración de nutrientes); $y$, por último, no tiene en cuenta el valor indicador de los táxones considerados (Moreno et al., 2006). Por tanto, la aplicación de un índice no aditivo que prima el valor indicador y de tolerancia frente a la riqueza, de resolución genérica (excepto briófitos) incluyendo un gran número de géneros, que calcula un valor indicador y otro de tolerancia para cada taxon a partir de las concentraciones de nutrientes, y que además presenta una respuesta al estado trófico similar o superior a la de índices europeos de resolución específica (tanto índices de macrófitos como de diatomeas), justifica en sí mismo su utilidad.

\section{Respuesta al estado trófico}

La respuesta del IVAM-FBL al estado trófico medido como concentración de nutrientes disueltos, ha resultado ser adecuada, destacando la alta co- 
rrelación obtenida con el ión fosfato $(r=-0.71$, $p<0.001)$. En Castilla-La Mancha se obtuvieron correlaciones superiores entre el IVAM-CLM $\mathrm{y}$ nutrientes $\left(r_{\text {fosfato }}=-0.77, r_{\text {amonio }}=-0.55\right.$, $r_{\text {nitrito }}=-0.63, r_{\text {nitrato }}=-0.39 ; p<0.001 ; r$ Spearman) (Moreno et al., 2005, 2006), debido probablemente a que en dicho estudio se incluyeron varias campañas de muestreo. En las tres cuencas catalanas estudiadas, se han realizado estudios previos de calidad del agua utilizando diatomeas (Gomà et al., 2004; datos de verano de 2002). Ello permite comparar la eficacia en la evaluación del estado trófico del IVAM-FBL frente a los índices de calidad de diatomeas. Así, Gomá et al. (2004) calcularon las correlaciones entre seis índices de diatomeas y la concentración de fosfato, obteniendo el coeficiente más alto con el índice IPS ( $r_{\text {pearson }}=-0.585, p<0.001$, $n=54$; datos transformados logarítmicamente), mientras que la Agencia Catalana del Agua (2003), entre tres índices de diatomeas probados, la correlación más alta fue obtenida con el índice $\operatorname{CEE~}\left(r_{\text {pearson }}=-0.539, p<0.05, n=212\right.$; datos transformados logarítmicamente). Por otra parte, es lógico dicho resultado, ya que el IPS o el CEE no son índices tróficos obtenidos exclusivamente a partir de las concentraciones de fosfato y amonio, sino que integran, además de nutrientes, información de otros parámetros. Para el nitrato, la mayor correlación fue encontrada con el índice de diatomeas IPS $\left(r_{\text {pearson }}=-0.332, p<0.05\right.$, $n=215$ ), una respuesta similar pero menos significativa que la encontrada con el IVAM-FBL ( $r=-0.31, p<0.01, n=68)$. En resumen, los valores del coeficiente de correlación entre los índices de diatomeas y el fosfato obtenidos en dichos estudios fueron menores que los encontrados para el IVAM-FBL y el IVAM-CLM, lo que indica una mayor eficacia del IVAM en detectar la eutrofización producida por fosfatos.

En las cuencas del Ter y Francolí de Cataluña se han aplicado algunos índices de macrófitos para evaluar la calidad, aunque solamente se han utilizado las macroalgas (ACA, 2003; datos de verano de 2002). Así, la mayor correlación con el fosfato la alcanzó el índice SAP (parámetro de regresión: $R^{2}=0.46$ ), mientras que fue muy baja para los otros dos índices aplicados, el SLA
$\left(R^{2}=0.15\right)$ y el E/P-I $\left(R^{2}=0.14\right)$. En el caso del IVAM-FBL, el valor de regresión calculado para la concentración de fosfato fue $\mathrm{R}^{2}=0.40$, similar aunque algo menor que el obtenido para el SAP. Esta ligera diferencia es contrarrestada por la ventaja de realizar un menor esfuerzo taxonómico en la aplicación del IVAM.

Los valores de correlación alcanzados entre el IVAM-FBL y el fosfato, fueron también superiores a los alcanzados en otros países por otros índices de macrófitos de resolución específica, como por ejemplo el MTR en el Reino Unido $\left(r_{\text {pearson }}=-0.549, p<0.001 ; n=2572\right.$; datos transformados), e incluso este índice no presentó una correlación significativa con el amonio (Dawson et al. 1999). En otro estudio realizado en el noreste de Francia, también se encontraron correlaciones entre el MTR y el fosfato menores que con el IVAM $\left(r_{\text {spearman }}=-0.567, p<0.05\right.$, $n=30)$, aunque sí fueron mayores con el amonio ( $r=-0.567, p<0.05)$ (Thiebaut et al., 2002).

Para la aplicación de estos índices europeos de diatomeas y de macrófitos, es necesaria la determinación específica de la mayoría de los táxones. El hecho de obtener una respuesta significativa al estado trófico con índices tanto específicos como genéricos, revela la gran ventaja de utilizar un índice genérico, ya que su obtención es mucho menos costosa, tanto en el tiempo empleado en el laboratorio como en la formación de personal capaz de aplicar los índices. En principio, parece que la pérdida de información debido a una determinación genérica de los organismos es suficientemente suplida por el hecho de utilizar varios grupos florísticos conjuntamente en la evaluación del estado trófico (algas, fanerógamas, briófitos, pteridófitos, líquenes). Incluir dicha diversidad es considerado fundamental en la obtención de una mejor respuesta a la eutrofización, en comparación con la utilización un solo grupo, por ejemplo las algas, incluso sin necesidad de alcanzar el nivel de resolución taxonómica de especie.

Finalmente, las perspectivas futuras de aplicación del IVAM pasan por considerar los siguientes aspectos: 1) recopilación de suficiente número de datos de varias zonas peninsulares (p. ej. norte de España) con el objeto de crear una tabla general de valores de tolerancia 
e indicador unificados para su aplicación en todo el territorio peninsular; 2) comparar los valores obtenidos por el IVAM con índices de diatomeas y otros índices de macrófitos, en una misma área de estudio y en una misma campaña de muestreo; 3) alcanzar la resolución genérica en musgos, hepáticas y líquenes.

\section{BIBLIOGRAFÍA}

AGENCIA CATALANA DEL AGUA (ACA). 2003. Anàlisi de la viabilitat i proposta d'indicadors fitobentònics de la qualitat de làigua per als cursos fluvials de Catalunya. Generalitat de Catalunya, Departament de Medi Ambient. 113 pp.

AFNOR. 2003. Qualité de l'eau-Détermination de l'indice biologique macrophytique en rivière (IBMR). AFNOR, NF T90-395. 28 pp.

ALBA-TERCEDOR, J., P. JÁIMEZ-CUÉLLAR, M. ÁlVAREZ, J. AVILÉS, N. BONADA, J. CASAS, A. MELLADO, M. ORTEGA, I. PARDO, N. PRAT, M. RIERADEVALL, S. ROBLES, C. E. SÁINZ-CANTERO, A. SÁNCHEZ-ORTEGA, M. L. SUÁREZ, M. TORO, M. R. VIDAL-ABARCA, S. VIVAS y C. ZAMORA-MUÑOZ. 2002. Caracterización del estado ecológico de los ríos mediterráneos ibéricos mediante el índice IBMWP (antes BMWP'). Limnetica, 21 (3-4): 175-185.

ALBA-TERCEDOR, J. y A. SÁNCHEZ-ORTEGA, A. 1988. Un método rápido y simple para evaluar la calidad biológica de las aguas corrientes basado en el de Hellawell (1978). Limnetica, 4: 51-56.

APHA. 1998. Standards methods for the examination of water and wastewater. American Public Health Association. $22^{\text {th }}$ edition. Washington D.C.

BONADA, N., N. PRAT, A. MUNNÉ, M. RIERADEVALL, J. ALBA-TERCEDOR, M. ALVAREZ, J. AVILÉS, J. CASAS, P. JÁIMEZ-CUÉLLAR, A. MELLADO, G. MOYA, I. PARDO, S. ROBLES, G. RAMÓN, M. L. SUÁREZ, M. TORO, R. VIDAL-ABARCA, S. VIVAS y C. ZAMORAMUÑOZ. 2002. Criterios para la selección de condiciones de referencia en los ríos mediterráneos. Resultados del proyecto GUADALMED. Limnetica, 21(3-4): 99-114.

CEMAGREF 1982. Etude des methods biologiques d'appréciation quantitative de la qualité des eaux. Rapport Q.E. Lyon, Agence de l'Eau Rhône-Méditerranée-Corse-Cemagref, Lyon, 218 pp.
DAWSON, F. H., J. R. NEWMAN, M. J. GRAVELLE, K. J. ROUEN \& P. HENVILLE. 1999. Assessment of the trophic status of rivers using macrophytes. Evaluation of the Mean Trophic Rank. Research and Development. Technical Report E39. Environment Agency, Bristol, 177 pp.

DELL'UOMO, A. 1991. Use of benthic algae for monitoring rivers in Italy. In: Use of benthic algae for monitoring rivers. B. A. Whitton, E. Rott \& G. Friederich (eds.): 129-137. Institut für Botanik, Universität Innsbruck.

DESCY, J. P. et M. COSTE. 1990. Utilisation des diatomées por l'évaluation de la qualité des eaux courantes. Rapport final. UNECED-Namur, CEMAGREF Bordeaux. 64 pp. + anexos.

GOMÀ, J., R. ORTIZ, J. CAMBRA \& L. ECTOR. 2004. Water quality evaluation in Catalonian mediterranean rivers using epilithic diatoms as bioindicators. Vie Milieu, 54(2-3): 81-90.

HAURY, J., M. C. PELTRE, S. MULLER, M. TREMOLIERES, J. BARBE, A. DAUTARTRE et M. GUERLESQUIN. 1996. Des indices macrophytiques pour estimer la qualité des cours d'eau français: premières propositions. Ecologie, 27: 233-244.

HAURY, J., M. C. PELTRE, S. MULLER, G. THIEBAUT, M. TREMOLIÈRES, B. DEMARS, J. BARBE, A. DAUTARTRE, H. DANIEL, I. BERNEZ, M. GUERLESQUIN et E. LAMBERT. 2000. Les macrophytes aquatiques bioindicateurs des systèmes lotiques - Intérêts et limites des indices macrophytiques. Synthèse bibliographique des principales approaches européennes puor le diagnostic biologique des cours déau. UMR INRAENSAR EQHC Rennes et Laboratoire de Phytoécologie Université de Metz. Agence de l'Eau Artois-Picardie, Etudes sur L'Eau en France, 87. Ministère de L'ecologie et du Développement Durable, France. 101 pp.

HAURY, J., M-C. PELTRE, M. TREMOLIERES, J. BARBE, G. THIE'BAUT, I. BERNEZ, H. DANIE, P. CHATENET, G. HAAN-ARCHIPOF, S. MULLER, A. DUTARTRE, C. LAPLACETREYTURE, A. CAZAUBON \& E. LAMBERTSERVIEN. 2006. A new method to assess water trophy and organic pollution-the Macrophyte Biological Index for Rivers (IBMR): its application to different types of rivers and pollution. Hydrobiologia, 570: 153-158.

MORENO, J. L., C. NAVARRO y J. DE LAS HERAS. 2005. Índice genérico de vegetación acuática 
(IVAM): propuesta de evaluación rápida del estado ecológico de los ríos ibéricos en aplicación de la Directiva Marco del Agua. Tecnología del Agua, 261: 48-53.

MORENO, J. L., C. NAVARRO y J. DE LAS HERAS. 2006. Propuesta de un índice de vegetación acuática (IVAM) para la evaluación del estado trófico de los ríos de Castilla-La Mancha: comparación con otros índices bióticos. Limnetica, 25(34): 211-228.

PRAT, N., A. MUNNÉ, C. SOLÁ, M. RIERADEVALL, N. BONADA y G. CHACÓN. 1999. La qualitat ecològica del Llobregat el Besòs i el Foix. Informe 1997. Diputació de Barcelona. Àrea de Medi Ambient (Estudis de la Qualitat Ecològica dels Rius; 6). Barcelona. 154 pp.

PRAT, N., MUNNÉ, A., SOLÀ, C., CASANOVASBERENGUER, R., VILA-ESCALÉ, M., BONADA, N., JUBANY, J., MIRALLES, M., PLANS, M., PUNTÍ, T. i M. RIERADEVALL. 2003. La qualitat ecològica del Llobregat, el Besòs, el Foix i la Tordera. Informe 2001. Diputació de Barcelona. Àrea de Medi Ambient (Estudis de la Qualitat Ecològica dels Rius; 11). 163 pp.

PRYGIEL, J. \& M. COSTE. 1999. Progress in the use of diatoms for monitoring rivers in France. En: Use of algae for monitoring rivers III. J. Prye- gel, B. A. Whitton B. A. \& J. Bukowska (eds.): 165-179. Agence de l'Eau Artois-Picardie, Douai, France.

SCHNEIDER, S. \& A. MELZER. 2003. The Trophic Index of Macrophytes (TIM)-a new tool for indicating the trophic state of running waters. Internat. Rev. Hydrobiol., 88: 49-67.

SLÁDECEK, V. \& A. SLADECKOVA. 1996. Atlas of aquatic organisms with respect to the water supply, surface waters and wastewater treatment plants. Ceská vedecko-technická vodohospodárská spolecnost, Praga. $351 \mathrm{pp}$.

SUÁREZ, M.L., A. MELLADO, M. M. SÁNCHEZMONTOYA y M. R. VIDAL-ABARCA. 2005. Propuesta de un índice de macrófitos (IM) para evaluar la calidad ecológica de los ríos de la cuenca del Segura. Limnetica, 24(3-4): 305-318.

THIEBAUT G., F. GUÉROLD \& S. MULLER. 2002. Are trophic and diversity indices based on macrophyte communities pertinent tools to monitor water quality? Water Research, 36: 3602-3610.

WEGL, R. 1983. Index für die Limnosaprobität. Wasser und Abwasser, 26: 1-175.

ZELINKA, M. und P. MARVAN. 1961. Zur Präzisierung der biologischen Klassification der Reinheit flissender Gewässer. Arch. Hydrobiol., 57: 389407. 